\title{
Improving the Dynamical Behaviour of a Laser Cutting Equipment by Using a Carbon Fibre Composite Main Structural Runway Frame
}

\author{
D. G. Vieira ${ }^{1 *} ;$ J. F. Meireles ${ }^{1} ;$ J. P. Nunes ${ }^{2}$ \\ ${ }^{1}$ Mechanical Engineering Dept., Minho University, Campus de Azurem, 4800-058 Guimaraes, \\ Portugal, \\ e-mail: a46979@alunos.uminho.pt \\ ${ }^{2}$ Polymer Engineering Dept., Minho University, Campus de Azurem, 4800-058 Guimaraes, \\ Portugal
}

Keywords: FEM; carbon fibre; composite, laser cutting equipment

\begin{abstract}
In order to improve the dynamic behaviour of an industrial laser cutting equipment a sandwich solution, using a carbon fibre reinforced polymer (CFRP) and polyester foam core, was implemented to construct its main runway structural frame, which supports the cutting head and major laser beam mirrors and lens.

Nowadays, the commercial competiveness of laser cutting equipments is considerable enhanced by their higher cutting speed and precision, as well as, cost. With the recent available higher power laser beam generators and swifter motors quicker and powerful cuts may be already done. However, at accelerations of 3 and 4 g's already enabled by linear motors, the lack of stiffness and high mass and consequent inertia of the traditional runway structural frames, made from steel and/or aluminium, do not allow achieving high required cutting precisions. Thus, the present study considered replacing those conventional materials by much lighter advanced CRFP composites to improve the dynamic performance of an existing laser cutting equipment.

Advanced numeric Finite Element Method (FEM) calculations by using the ANSYS package software were made to verify the static and dynamic behaviours of the new composite structural frame and compare them to simulations made with the currently used steel solution.

The composite structural frame processing method has been also studied and defined in this work. Furthermore, the composite laminate has been optimised by defining the better number of stacking layers and fibre orientations to be used, as well as, the foam core thickness. The failure of the new sandwich structural composite runway frame has been verified through the Tsai-Wu criterion. Finally, an economic analysis of the viability of the new composite solution adopted will be also presented.
\end{abstract}

\section{Introduction}

Nowadays we live in a global market era that forces machine tooling industries to build not only much higher performance equipments at low cost but also in short conception time. High precision tooling manufacturing industry, such as the manufactures of laser beam cutting equipments, is particularly affected by such competiveness pressure.

Therefore, the use of materials presenting higher "mechanical performance/weight" ratios, such as carbon fibres, becomes an asset. The easy processing of that use low density polymer matrix composites reinforced with this type of high performance fibres is currently spreading the use of these materials in many high advanced application fields [1]. In fact, carbon fibre reinforced polymer matrix composites (CFRP) are presently quite use in many advanced applications (such as, sport, space and military aircraft industries) and start now to be applied in civil aviation and manufacturing industries.

For any application, replacing traditional materials by new ones involves, at least, the following relevant engineering tasks [2]:

1. Selecting materials able to better accomplish the main product or application [2]. 
2. Redesign the existing product to achieve higher performance and reliability with lower cost and weight.

To achieve the best result in the above mentioned $2^{\text {nd }}$ task large work must be carried out [3]. The Finite element method (FEM) is one of the best ways to quickly achieve optimal results in the design and/or redesign of a composite product [2]. The diagram in Figure 1, shows the main steps needed to implement a good engineering redesign of a composite product by considering the loading and boundary working conditions that the structural product is submitted.

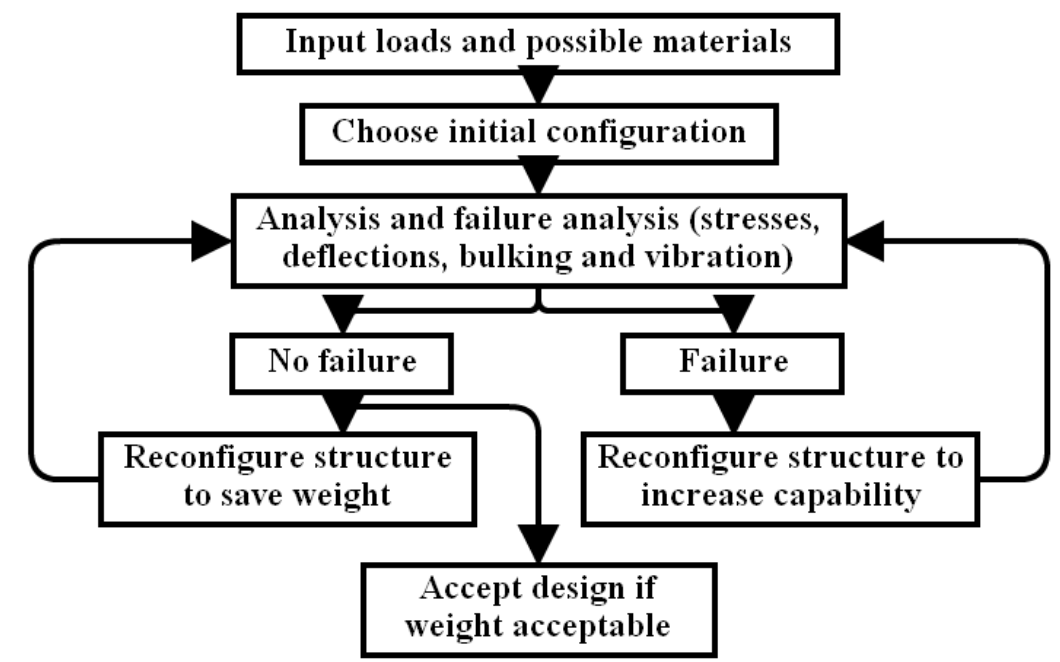

Fig. 1 - Stages in the structures execution [3]

\section{Materials Selection and Preliminary studies}

A sandwich structural wall using a carbon fibre reinforced epoxy matrix composite (CFRP) in both outside surfaces and a polyester foam as internal core was selected to produce by vacuum infusion the new main framework runway beam of the laser cutting machine. To quickly optimise the new composite layered wall, the Steiner's theorem was used to determine, in terms of stiffness, an equivalent composite structural wall to that one currently made in steel [4].

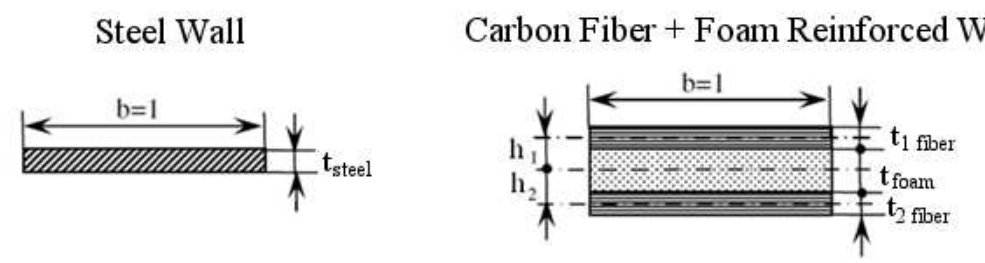

Fig. 2 - Diagram of a sandwich type composite material structure

By considering Fig. 2, both structural walls present equivalent present the same stiffness, when:

$$
E_{\text {steel }} \times I_{\text {steel }}=E_{\text {steel }} \times \frac{t_{\text {steel }}^{3}}{12} \times b
$$

where $E, I, t$ and $b$ are the materials Young' moduli and wall inertia moment, thicknesses and width, respectively.

Thus, the equivalent sandwich composite wall in terms of stiffness may be calculated through:

$$
E_{\text {fiber }} \times\left(\frac{t_{1 \text { fiber }}^{3}}{12} \times b+t_{1 \text { fiber }} \times b \times\left(h_{1}^{2}\right)\right)+E_{\text {foam }} \times \frac{t_{\text {foam }}^{3}}{12} \times b+E_{\text {fiber }} \times\left(\frac{t_{2 \text { fiber }}^{3}}{12} \times b+t_{2 \text { fiber }} \times b \times\left(h_{2}^{2}\right)\right)
$$

where:

$$
h_{1}=\frac{t_{1 \text { fiber }}+t_{\text {foam }}}{2}
$$


From the above calculations a sandwich composite symmetrical laminate $\left[\left(0^{\circ}, 90^{\circ}, \pm 45^{\circ}\right)_{5} \text {, foam }\right]_{\mathrm{S}}$, with an approximated total and polyester foam thickness of $7 \mathrm{~mm}$ and $3 \mathrm{~mm}$, respectively, was selected for the framework beam structural wall.

The interactive Tsai-Wu criterion, shown in Eq. 5, was used to predict the failure of layers in any different direction $[4,5]$ :

$$
F_{1} \cdot \sigma_{1}+F_{2} \cdot \sigma_{1}+F_{11} \cdot \sigma_{1}^{2}+F_{22} \cdot \sigma_{2}^{2}+F_{66} \cdot \tau_{12}^{2}+2 \cdot F_{12} \cdot \sigma_{1} \cdot \sigma_{2}=1
$$

where $\sigma_{1}$ and $\sigma_{2}$ are the normal stresses in the fibre and transverse to the carbon fibre directions, respectively, $\tau_{12}$ is the shear stress and $F_{1}, F_{2}, F_{11}, F_{22}, F_{66}, F_{12}$ are layer characteristics experimentally determined by mechanical testing [4].

\section{Studied Case}

The laser cutting machine has four linear motors: two on the ends of the runway beam frame and two mounted in the cutting head supporting car. The high concentrated laser beam passes directly through the focusing lens mounted on the car that moves in the axial direction by slipping in the runway framework beam, Fig. 3.

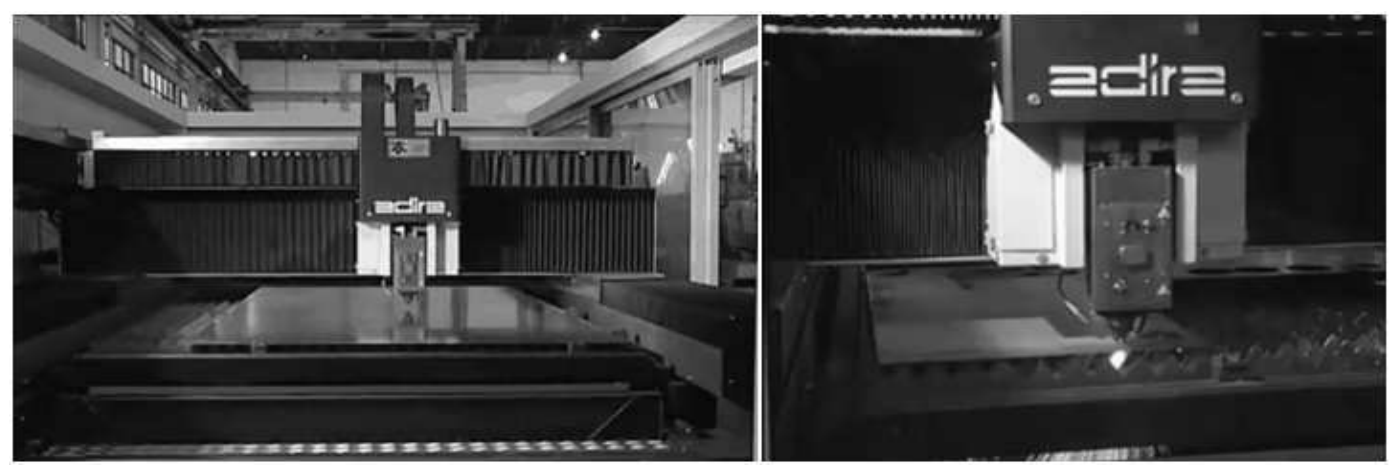

Fig. 3 - Framework runway beam of the laser machine during a cut.

By ensuring the main movements of the equipment cutting head the framework runway beam must be considered the key component of the machine. This component must not only withstand a high level of mechanical loads but also ensure a very low level of deformations because they are reflected in laser beam deviations and so in cut-off and subsequent loss of cutting machine precision. Thus, as the maximum equipment cutting speed is limited by the deformation suffered the final performance of the machine is seriously affected by this matter.

Fig. 4 shows the steel framework runway beam that is currently used in the laser cutting machine illustrated in Fig. 3. As can be seen the external frame beam box is reinforced by various internal steel components.

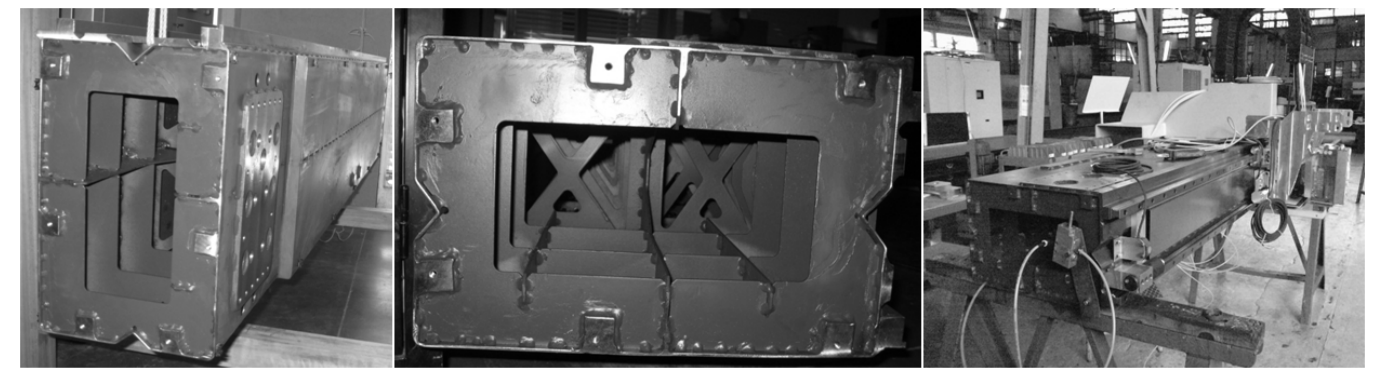

Fig. 4 - Details of the construction of the steel beam currently produced.

In fact, the current main framework runway beam is manufactured using two steel box girder type substructures internally supported in diverse steel plates, some of them having the "X", "C" shapes that may be seen in Fig. 4. The steel box girder that supports the cutting head car slipping guides uses also steel $\mathrm{V}$-shape plates to allow reinforcing the wall where the slipping guides are mounted. All these plates are assembled and connected together by using multi-point and also continuous welding methods. 


\section{Composite Configuration}

All composite components for the renewed main framework runway beam were designed (four components form the beam) in order to be symmetrical, capable of being processed by infusion and, most of all, to allow using the minimum number of moulds in the entire structure manufacturing. This will allow reducing the investment in moulds that largely affects the final price of a composite component.

The shape of the carbon fibre reinforced epoxy (CFRP) framework beam has also been designed to allow bonding all components by using a structural epoxy adhesive. Therefore, all components present adequate surface areas to allow good bonding and also enable their positions with good accuracy for the assembly of the beam. Fig. 5 shows the CRRP composite component \#1. Twentyeight components of this type 1 will be needed to manufacture the new composite framework beam. The X-shape that included in the component allows improving considerably its torsional rigidity and behaviour without increase the thickness and weight of the part significantly. All concordance radii were made to be greater than $5 \mathrm{~mm}$ to facilitate the placement of carbon fibre fabrics in the mould and manufacturing.
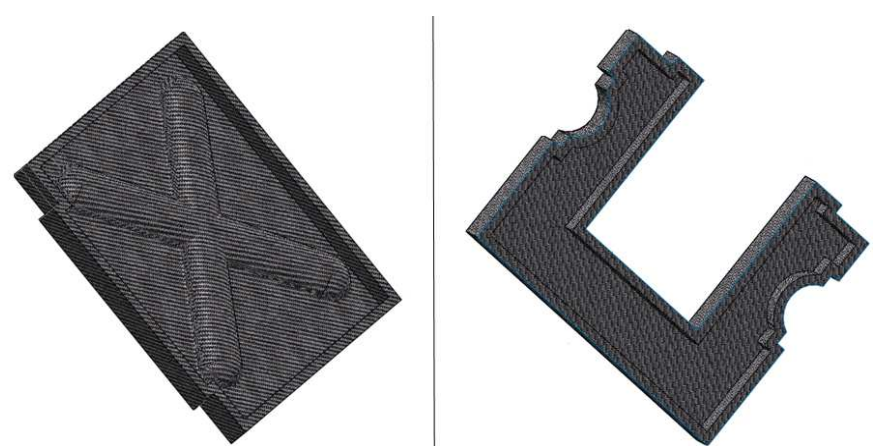

Fig. 5 - Composite component \#1 Fig. 6 - Composite component \#2

Fig. 6 illustrates the CRRP composite component \#2. Each composite framework beam is composed by twelve components of this type. A C-shape has been chosen for this part in order to allow increasing the overall structure rigidity in the framework beam extremities and maintain the access openings needed to assemble equipments.

The composite part shown in Fig. 7 a) was also design with a U-shape to increase the rigidity of the structure in a specific region where the framework beam do not present free space to use greater reinforcing composite components. The lack of free space in that specific framework region is related we the need of having a local unobstructed area to allow accessing equipments assembled to the frame beam. Such U-shape CRRP reinforcing composite component was design to be glued to the other components, in particular to C-shape type composite component \#2 shown in Fig. 6. Eight composite components of this type are used in each framework beam.

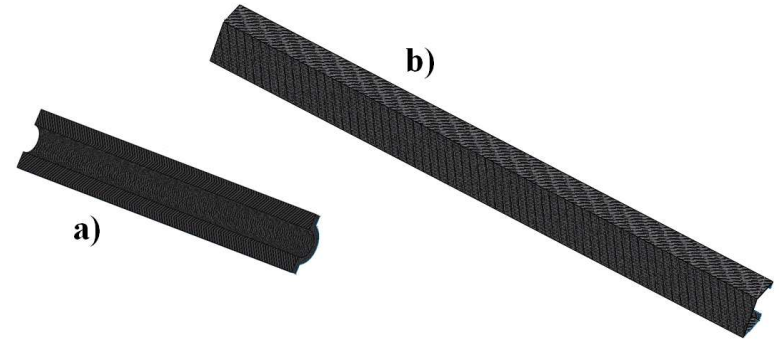

Fig. 7 - a) Composite component \#3; b) Composite component \#4

Finally, the composite reinforcing part \#3 shown in Fig. 7 b) is used in the framework beam outer shells. All previous composite reinforcing parts are glued to the outlying areas of this last component and two of them are applied in each framework beam.

Only the length of this last composite reinforcing component must be changed when smaller framework beams are manufactured. Thus, it should be emphasise that all CRRP composite components were designed for being used in various machine models currently manufactured by the 
company. Therefore, the same framework beam moulds may be used to produce of all different machine size range. In some cases only changes the number of components used in the manufacture.

The total weight of the bigger framework composite beam structure made is $62 \mathrm{~kg}$, which consists in reducing the weight of the currently produced steel beam frame by $64 \%$.

\section{Mechanical and Economic Analysis}

FEM was used to validate the mechanical response of the new designed CFRP framework beam in the working and boundary conditions. The most demanding requirement concerns the maximum allowable deformation the framework beam structure may reach when statically charged. In this case, in accordance to the design requirements the maximum allowable deviation that may be produced in the optical cutting laser beam cannot overpass $0.018^{\circ}$. For the new composite sandwich framework proposed in this work, having CFRP outside layers and a polyester foam core, a maximum deviation of $0.017^{\circ}$ was obtained for the optical cutting laser beam, which fulfils the design requirement.

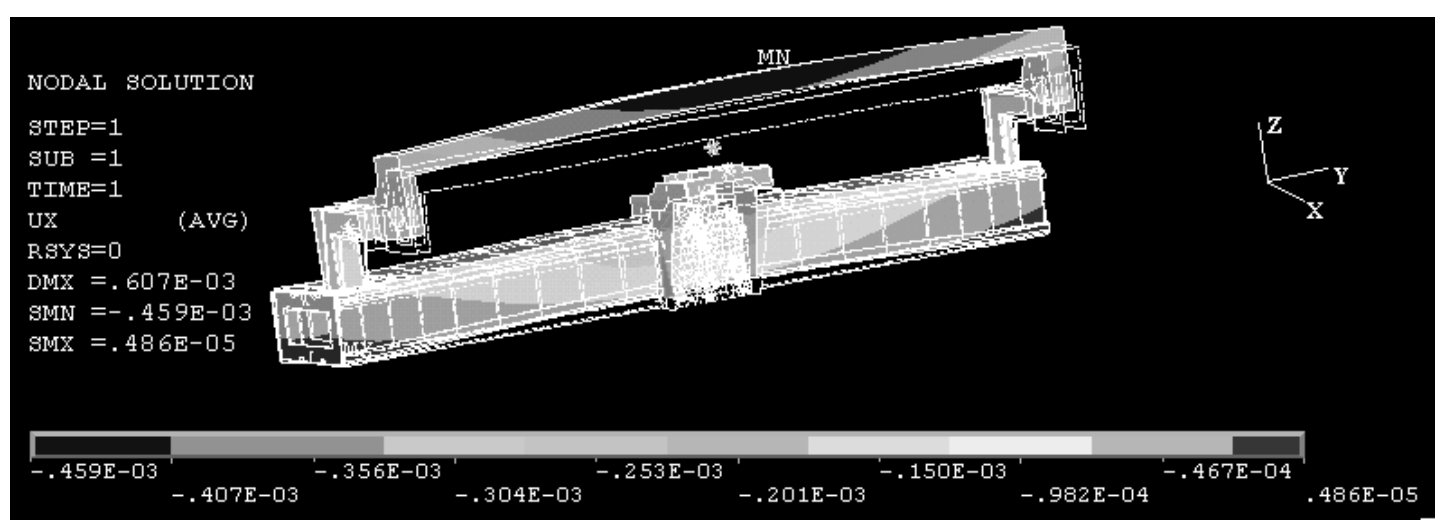

Fig. 8 - Dispalcement in XX direction [m]

Fig. 8 shows the deformation of the structure in $\mathrm{XX}$ direction. The beam extremities present the locals where the structure was more deformed. On the other hand, the middle of the beam presents the locals where the structure suffers lower deformations.

Concerning the dynamic analysis and not considering the rigid vibration modes (6 first modes), the resonance on the composite and steel framework beams occurs at frequencies shown in Table 1. The table only shows some of the vibration modes. The simulation was performed on the 0 to $500 \mathrm{~Hz}$ range. In terms of dynamic results, it were found 7 and 47 vibration modes in the cases of the composite and currently produced steel framework beams, respectively. As expected, it may be also seen that the new composite framework beam present a much better dynamic behaviour than the currently used steel structure.

Table 1 - Natural frequencies and vibration modes of composite and steel structures

\begin{tabular}{ccc|ccc}
\hline $\begin{array}{c}\text { Vibration } \\
\text { Mode }\end{array}$ & $\begin{array}{c}\text { Natural } \\
\text { Frequency [Hz] }\end{array}$ & $\begin{array}{c}\text { Vibration } \\
\text { Mode Type }\end{array}$ & $\begin{array}{c}\text { Vibration } \\
\text { Mode }\end{array}$ & $\begin{array}{c}\text { Natural } \\
\text { Frequency [Hz] }\end{array}$ & $\begin{array}{c}\text { Vibration } \\
\text { Mode Type }\end{array}$ \\
\hline \multicolumn{2}{c|}{ Composite Structure } & \multicolumn{3}{c}{ Steel Structure } \\
\hline Mode 7 & 153,15 & Flexion & Mode 7 & 18,26 & Flexion \\
Mode 8 & 202,64 & Flexion & Mode 8 & 20,13 & Flexion \\
Mode 9 & 253,96 & Torsion & Mode 9 & 125,36 & Flexion \\
Mode 10 & 321,36 & Flexion & Mode 10 & 208,79 & Flexion \\
Mode 11 & 390,70 & Flexion & Mode 11 & 209,13 & Flexion \\
Mode 12 & 466,83 & Torsion & Mode 12 & 209,38 & Flexion \\
\hline
\end{tabular}

Regarding costs involved, the manufacture of the new proposed composite framework sandwich structure, using CFRP outside layers involving a polyester foam core, seems present much higher cost than the one associated to the production of the currently produced steel structure. The initial 
predictions done make expect that the new composite framework beam could be about 6.6 times more costly than the currently steel one used. However, deeper studies concerning the impact caused in the market by the launch of the new laser cutting machine, that presents much higher performance in terms of speed and precision, and also the benefits for the manufacturing company that may result from the technological advanced knowledge acquired should be made in order to take definitive conclusions about the economic final profits and losses obtained.

\section{Conclusions}

In this work a totally new composite main runway frame beams were developed to replace the steel currently used ones in order to improve significantly the performance of laser cutting machines that are manufactured by a company. The static mechanical behaviour of the new composite framework structure was validated by FEM. Dynamically, the behaviour of the new composite framework beam behaviour should be revised and optimised because it has demonstrated, when having all its components attached, to present some worrying behaviour at frequencies in the possible machine working range. However, the new composite framework beam has clearly shown to present much better dynamic behaviour than the currently one made of steel.

This new type of materials and technologies could bring benefits to the company, not only in terms of the better performance of the laser cutting equipments but also because of technological advantage it may take over all other market competitors.

\section{Acknowledgements}

The authors acknowledge Adira SA for the opportunity given to study a real structural frame coupled on a high-end tool-machine.

\section{References}

[1] M. F. S. F. Moura, A. B. Morais, A. G. Magalhães, Materiais Compósitos: Materiais, Fabrico e Comportamento Mecânico, Publindústria, Porto/Portugal, 2005.

[2] S. K. Mazumdar,Composites Manufacturing : Materials, Product, and Process Engineering. CRC Press LLC, New York, 2002.

[3] R. M. Jones, Mechanics of Composite Materials, $2^{\text {nd }}$ edition Taylor \& Francis eds, New York, 1999.

[4] J. P. Nunes, A Study of the Processing and Properties of Sheet Molding Compounds and Unidirectional Carbon Fibre Towpregs, PhD Thesis, Minho University, Guimarães/Portugal, 1998.

[5] L. P. Kollar, G. S. Springer, Mechanics of Composite Structures, Cambridge University Press, New York, 2003. 